\title{
Utilização da Estratégia de Atenção Integrada às Doenças Prevalentes na Infância
}

\author{
Ingrid Letícia Fernandes dos Santos ${ }^{1}$, \\ Maria Aparecida Munhoz Gaíva², \\ Ana Karina Marques Salge ${ }^{3}$
}

\section{RESUMO}

Estudo que objetivou descrever a utilização da Estratégia de Atenção Integrada às Doenças Prevalentes na Infância (AIDPI) por médicos e enfermeiros que atuavam na Estratégia Saúde da Família em capital do centro-oeste brasileiro. Estudo descritivo desenvolvido com 50 médicos e 51 enfermeiros que trabalhavam em unidades de saúde da família. Os dados foram coletados utilizando-se um questionário. A maioria dos pesquisados era do sexo feminino $(69,3 \%)$, com três a cinco anos de formados $(32,7 \%)$, sendo que $50,5 \%$ demonstrou conhecer o conteúdo sobre a estratégia, aprendido durante a graduação. Cerca de $44,6 \%$ dos profissionais utiliza parcialmente a estratégia, sendo que o elemento mais usado por eles é a avaliação de crescimento e anemia $(25,4 \%)$. Os resultados encontrados são preocupantes, pois a não utilização de todos os componentes da AIDPI pode refletir na impossibilidade de detecção de doenças tratáveis na atenção primária, aumentando o número de internações hospitalares evitáveis.

Descritores: Atenção Integrada às Doenças Prevalentes na Infância; Atenção Primária em Saúde; Enfermagem Pediátrica; Saúde da Criança.

\footnotetext{
${ }^{1}$ Enfermeira, Mestre em Enfermagem. Professora do Centro Universitário de Várzea Grande. Várzea Grande, MT, Brasil. E-mail: i.leticiafs@gmail.com.

${ }^{2}$ Enfermeira, Doutora em Enfermagem em Saúde Pública. Professora Associada da Faculdade de Enfermagem da Universidade Federal de Mato Grosso. Cuiabá, MT, Brasil. E-mail: mamgaiva@yahoo.com.br.

${ }^{3}$ Enfermeira, Doutora em Patologia. Professora Associada da Faculdade de Enfermagem da Universidade Federal de Goiás. Goiânia, GO, Brasil. Email: anasalge@gmail.com.
}

Artigo recebido: 28/07/2017.

Artigo aprovado: 28/03/2018.

Artigo publicado: 28/11/2018.

\section{Como citar esse artigo:}

Santos ILF, Gaíva MAM, Salge AKM. Utilização da Estratégia de Atenção Integrada às Doenças Prevalentes na Infância. Rev. Eletr. Enf. [Internet]. 2018 [acesso em: ];20:v20a26. Disponível em: https://doi.org/10.5216/ree.v20.49053. 


\section{INTRODUÇÃO}

No início da década dos anos 90, as precárias condições de saúde da população infantil em todo mundo fizeram com que a Organização Mundial da Saúde (OMS), a Organização Pan-Americana de Saúde (OPAS) e o Fundo das Nações Unidas para a Infância (UNICEF) propusessem a Estratégia de Atenção Integrada às Doenças Prevalentes na Infância (AIDPI) ${ }^{(1)}$. O Brasil adotou a estratégia, oficialmente, em 1995, contudo, a incorporação da metodologia de atendimento às ações da Estratégia Saúde da Família só aconteceu em 1996, quando foram feitas as adaptações dos protocolos à realidade epidemiológica brasileira.

A AIDPI possui como objetivos: responder às necessidades de saúde da população infantil; fortalecer o enfoque integrado de atenção à saúde da criança; aplicar medidas de prevenção; estimular atividades de promoção da saúde; melhorar a eficiência e qualidade da atenção; atuar como porta de entrada para a deteç̧ão e tratamento de outros problemas de saúde, de forma a reduzir a mortalidade infantil(2). Essa estratégia propõe um conjunto de ações preventivas e curativas que inclui a educação em saúde, principalmente para aconselhamento na recuperação nutricional, incentivo ao aleitamento materno e imunização, contribuindo para a melhoria das condições de saúde das crianças.

A Organização Mundial de Saúde (OMS) não especifica quais profissionais devem aplicar a AIDPI, no entanto, suas normas foram elaboradas para utilização, preferencialmente, em unidades de atenção primária à saúde. No Brasil, os enfermeiros e médicos, em especial os que atuam na Estratégia Saúde da Família (ESF) devem ser capacitados para desenvolver tal prática.

A importância da AIDPI tem sido demonstrada pelas mudanças ocorridas nos indicadores de saúde em países que a adotaram. Países como Afeganistão, Yemen, Gana, Marrocos, Bangladesh, Uganda, Tanzânia e Botswana apresentaram melhoras significativas na qualidade da atenção à saúde das crianças, bem como a redução de hospitalizações e do índice de mortalidade infantil(3-7).

No Brasil, apesar do decréscimo acentuado da mortalidade infantil nas últimas três décadas, em especial nas regiões mais pobres do país ${ }^{(8)}$, não há resultados de pesquisas demostrando a relação entre a implantação da AIDPI e a redução das mortes infantis.

A despeito do importante impacto produzido pela implantação da estratégia, muitos países enfrentaram dificuldades para sua implementação, entre elas, baixa cobertura de treinamento inicial para os profissionais de saúde, falta de medicamentos e suprimentos essenciais, falta de tutoria e de cursos de atualização e supervisão de apoio regular ${ }^{(4)}$, financiamento inadequado e o alto custo dos cursos de treinamento ${ }^{(7)}$. Ainda para os autores do estudo referido, essas dificuldades poderiam ser enfrentadas aumentando o número de profissionais de saúde treinados na estratégia AIDPI e a extensão do treinamento para todos os profissionais de saúde, inclusive os de nível médio.

Texto que discutiu os 20 anos de implementação da AIDPI e seus impactos na África do Sul apontou que este país teve alguns sucessos na implementação da estratégia, em especial quanto a cobertura de treinamento para profissionais da atenção primária à saúde. No entanto, a prática limitada da AIDIP por profissionais treinados e a baixa adesão dos médicos às suas diretrizes levaram a uma implementação fraca da estratégia e impacto inadequado na melhoria saúde infantil no país ${ }^{(9)}$. 
A implantação e implementação da AIDPI tem ocorrido de forma parcial em muitos países ${ }^{(7,9)}$. No Brasil, apesar de não se ter pesquisas atuais sobre a implementação da AIDIP, estudo realizado em meados dos anos 2000 evidenciou que nas regiões de maior concentração de profissionais de saúde, a estratégia não havia sido implementada ${ }^{(10)}$. Não obstante os profissionais de enfermagem reconhecerem a importância da estratégia, sua aplicação tem sido limitada ao módulo de avaliação dos sinais e sintomas ${ }^{(11)}$.

Por sua vez, pesquisa realizada na cidade de Fortaleza-Brasil e em Lima-Peru, com gestores de saúde e facilitadores da estratégia, também apontou o predomínio na implementação do componente clínico, em detrimento dos componentes comunitário e de serviços de saúde. Os resultados mostraram que no Peru a estratégia foi adotada pelo governo e incluída nas políticas de saúde infantil, enquanto que no Brasil, ela ainda não está estabelecida nem em nível de país nem de Estado, além disso, a sistemática da estratégia não foi incorporada como rotina nos cuidados de saúde infantil e o governo brasileiro reduziu os investimetnos na formação dos profissionais para a estratégia ${ }^{(12)}$.

Embora ainda careça de pesquisas que comprovem o decrescente uso da AIDPI em nosso país, os poucos estudos mais atuais mostram limitações em sua implementação. A esse respeito, cabe salientar que, a AIDPI não é um projeto acabado e, além de políticas e reforços nas linhas estratégicas de promoção e prevenção à saúde infantil, necessita de maiores investimentos na capacitação dos profissionais de saúde para a sua efetiva implementação.

A AIDPI se configura como estratégia que reforça o conceito de integralidade da assistência à criança, contribuindo para a resolutividade no primeiro nível de atenção, além de ser uma ferramenta que apoia a sistematização da assistência infantil, portanto, a sua utilização pelos profissionais da saúde da família é fundamental para a assistência e promoção da saúde da criança.

Tendo em vista a escassez de estudos atuais sobre a utilização da AIDPI na realidade brasileira e que a única pesquisa desenvolvida na região centro-oeste sinalizou a utilização parcial da estratégia pelos profissionais da saúde da família, além da ausência de informações oficiais sobre como se encontra o processo de implementação da AIDPI no Estado de Mato Grosso e na capital, Cuiabá, este estudo justifica-se, e contribui para mapear essa situação no contexto brasileiro, além de oferecer subsídios para a avaliação das ações desenvolvidas pelos profissionais de saúde de forma a qualificar a assistência à saúde da criança na atenção primária.

Assim, o presente estudo objetivou descrever a utilização da Estratégia de Atenção Integrada às Doenças Prevalentes na Infância (AIDPI) por médicos e enfermeiros que atuavam na Estratégia Saúde da Família em uma capital do centro-oeste brasileiro.

\section{MÉTODO}

Estudo descritivo de abordagem quantitativa sobre a utilização da estratégia AIDPI por enfermeiros e médicos que atuavam nas unidades de saúde da família (USF) de uma capital do centro-oeste brasileiro. A investigação foi desenvolvida nas USF, em razão da aplicação da AIDPI estar vinculada a este espaço de atuação dos profissionais da saúde.

A pesquisa foi realizada em Cuiabá, capital do Estado do Mato Grosso/Brasil e que possuía em 2013, à época da coleta de dados da pesquisa, 62 equipes de saúde da família, estas sendo compostas por uma equipe 
multidisciplinar (médico, enfermeiro, técnicos/auxiliares de enfermagem e agentes comunitários de saúde), atuando na zona urbana da capital.

Considerou-se como população deste estudo, 109 profissionais, dentre estes, 58 enfermeiros e 51 médicos. A escolha dos participantes foi intencional, sendo incluídos no estudo os profissionais que estavam em atividade nas equipes de saúde da família no período de coleta de dados, perfazendo um total de 101 profissionais, 50 médicos e 51 enfermeiros. Cabe ressaltar que nenhum profissional se recusou a participar da pesquisa.

Para coleta de dados foi utilizado um questionário elaborado pelas pesquisadoras, o qual foi submetido a um pré-teste em outro município, para detectar e solucionar possíveis problemas ou erros referentes à clareza das questões, preenchimento do instrumento ou outros que pudessem surgir durante a pesquisa. Os dados foram coletados pelo pesquisador responsável e por estudantes de graduação em enfermagem, previamente treinados, entre março a maio de 2013.

No que tange ao questionário, cabe salientar que o mesmo foi composto por perguntas abertas e fechadas, com dados sobre a identificação do profissional e da USF que atuava, dados sobre a formação profissional dos participantes, capacitação recebida sobre a estratégia e questões acerca da utilização da AIDPI, tomando por base os manuais de curso de capacitação sobre a estratégia do Ministério da Saúde.

Para analisar a utilização da AIDPI pelos profissionais, foi considerada a metodologia do processo de atenção integrada de casos de AIDPI, proposta pela OPAS 2009(2), que inclui os seguintes elementos: avaliar a criança (verificação dos sinais e sintomas das doenças prevalentes), classificar a doença, determinar o tratamento, tratar segundo a classificação, aconselhar a mãe/responsável e agendar retorno. Quanto à categorização realizada para orientar o processo analítico, foram classificadas como utiliza totalmente, as respostas obtidas que contemplavam todos esses elementos.

Os dados foram processados com os recursos do software estatístico EPI-Info na versão 3.5.2. Para análise, foi utilizada a estatística descritiva, através da avaliação de percentual da frequência de respostas, sendo os resultados apresentados em tabelas.

O presente estudo respeitou todos os preceitos éticos da Resolução 466/2012 do Conselho Nacional de Saúde e foi aprovado por Comitê de Ética em Pesquisa sob o parecer no 194.315.

\section{RESULTADOS}

Os resultados advindos do processo de pesquisa permitem afirmar que, dentre os participantes da pesquisa, (69,3\%) era do sexo feminino, com graduação em enfermagem (50,5\%), sendo que $47,5 \%$ relatou ter-se graduado nos últimos cinco anos. Sobre a pós-graduação, 70,3\% disse possuir algum tipo de curso lato sensu, sendo $41,6 \%$ na área da saúde da família. No que se refere ao tempo de atuação na ESF, $29,7 \%$ dos profissionais atuavam entre três a cinco anos (Tabela 1 ).

A Tabela 2 apresenta as características da formação e capacitação dos profissionais em AIDIP. Dos 101 pesquisados, 50,5\% afirmaram que a estratégia foi abordada durante a graduação, desses, 37,3\% consideraram que tal abordagem foi boa. Dentre os respondentes, a maior parte dos profissionais afirma não ter sido capacitada para desenvolver a estratégia. 
Tabela 1: Perfil dos enfermeiros e médicos atuantes nas Equipes de saúde da Família de Cuiabá, Mato Grosso, Brasil, 2013. ( $N=101$ )

\begin{tabular}{|c|c|c|c|}
\hline & Variáveis & $f$ & $\%$ \\
\hline \multicolumn{4}{|l|}{ Sexo } \\
\hline Feminino & & 70 & 69,3 \\
\hline Masculino & & 31 & 30,7 \\
\hline \multicolumn{4}{|l|}{ Graduação } \\
\hline Enfermagem & & 51 & 50,5 \\
\hline Medicina & & 50 & 49,5 \\
\hline \multicolumn{4}{|l|}{ Tempo de graduado } \\
\hline Menor de 1 ano & & 6 & 6,0 \\
\hline 1 a 2 anos & & 9 & 8,9 \\
\hline 3 a 5 anos & & 33 & 32,7 \\
\hline 6 a 10 anos & & 27 & 26,7 \\
\hline Acima de 10 anos & & 26 & 25,7 \\
\hline \multicolumn{4}{|c|}{ Pós-graduação lato sensu } \\
\hline Sim & & 71 & 70,3 \\
\hline Não & & 24 & 23,8 \\
\hline Andamento & & 6 & 5,9 \\
\hline \multicolumn{4}{|l|}{ Área da pós-graduação } \\
\hline Saúde da família & & 32 & 41,6 \\
\hline Saúde pública & & 15 & 19,5 \\
\hline Saúde da criança & & 2 & 2,6 \\
\hline Outras & & 28 & 36,3 \\
\hline \multicolumn{4}{|c|}{ Tempo de atuação na ESF* } \\
\hline Menor de 1 ano & & 15 & 14,8 \\
\hline 1 a 2 anos & & 24 & 23,8 \\
\hline 3 a 5 anos & & 30 & 29,7 \\
\hline 6 a 10 anos & & 19 & 18,8 \\
\hline Acima de 10 anos & & 13 & 12,9 \\
\hline
\end{tabular}

* ESF: estratégia saúde da família. Fonte: dados da pesquisa.

Tabela 2: Abordagem da Atenção Integrada às Doenças Prevalentes na Infância durante a formação dos profissionais enfermeiros e médicos atuantes nas equipes de saúde da família de Cuiabá, Mato Grosso, Brasil, 2013. (N=101).

\begin{tabular}{lcc}
\hline \multicolumn{1}{c}{ Variáveis } & $\mathbf{f}$ & $\%$ \\
\hline Abordagem da AIDPI durante a graduação & 51 & 50,5 \\
Sim & 50 & 49,5 \\
Não & & 37,3 \\
Opinião sobre a qualidade do conteúdo AIDPI ofertado durante a graduação & 19 & 33,3 \\
Bom & 17 & 29,4 \\
Muito bom & 15 & 26,7 \\
Insuficiente & & 73,3 \\
Capacitação em AIDPI & 27 & 74 \\
Sim & & 2 \\
Não & & 2
\end{tabular}

* AIDPI: Atenção Integrada às Doenças Prevalentes na Infância.

Do total dos profissionais entrevistados, $44,6 \%$ relatou utilizar parcialmente a AIDPI. Dentre os elementos da estratégia mais utilizados por eles estão a avaliação do crescimento e anemia $(25,4 \%)$, alimentação (23,2\%). Os motivos destacados para a ausência da utilização da AIDPI foram, não possuir capacitação (48,8\%), e desconhecimento da estratégia $(31,7 \%)$, conforme Tabela 3. 
Tabela 3: Utilização da Atenção Integrada às Doenças Prevalentes na Infância pelos profissionais atuantes nas equipes de saúde da família de Cuiabá, Mato Grosso, Brasil, 2013.

\begin{tabular}{|c|c|c|}
\hline Variáveis & $f$ & $\%$ \\
\hline \multicolumn{3}{|l|}{ Como os profissionais utilizam AIDPI (N=101) } \\
\hline Totalmente ${ }^{1}$ & 18 & 17,8 \\
\hline Parcialmente & 45 & 44,6 \\
\hline Não utiliza & 38 & 37,6 \\
\hline \multicolumn{3}{|l|}{ Elementos da AIDPI mais utilizados ${ }^{2}(\mathrm{~N}=138)$} \\
\hline Avaliação de crescimento e anemia & 35 & 25,4 \\
\hline Avaliação da alimentação & 32 & 23,1 \\
\hline Avaliação dos sinais de perigo & 31 & 22,5 \\
\hline Classificação das doenças & 24 & 17,4 \\
\hline Prescrição das medicações & 16 & 11,6 \\
\hline \multicolumn{3}{|l|}{ Motivos para a não aplicação da $\mathrm{AIDPI}^{3}$ ( $\mathrm{N}=38$ ) } \\
\hline Não possuir capacitação & 20 & 52,7 \\
\hline Desconhecimento da estratégia & 13 & 34,2 \\
\hline Não saber utilizá-la & 5 & 13,1 \\
\hline \multicolumn{3}{|l|}{ Outras estratégias utilizadas para avaliar a criança ${ }^{4}(N=57)$} \\
\hline Crescimento e desenvolvimento & 32 & 56,1 \\
\hline Programa de suplementação de ferro & 18 & 31,6 \\
\hline Protocolos de atendimentos ambulatoriais & 7 & 12,3 \\
\hline \multicolumn{3}{|l|}{ Legenda: } \\
\hline \multicolumn{3}{|c|}{$\begin{array}{l}{ }^{1} \text { Foram classificadas como totalmente, as respostas que contemplaram todos os elementos: avaliar a criança (verificação dos sinais e sintomas das } \\
\text { doenças prevalentes), classificar a doença, determinar o tratamento, tratar segundo a classificação, aconselhar a mãe/responsável e agendar retorno. }\end{array}$} \\
\hline \multicolumn{3}{|c|}{$\begin{array}{l}{ }^{2} \mathrm{~A} \text { questão sobre os elementos mais utilizados da estratégia foi direcionada aos profissionais que declararam utilizar parcialmente a estratégia e eles } \\
\text { podiam dar mais de uma resposta, portanto o } \mathrm{N} \text { correspondeu ao total das respostas (138). }\end{array}$} \\
\hline \multicolumn{3}{|c|}{${ }^{3} \mathrm{~A}$ questão sobre os motivos para a não aplicação da AIDPI era apenas para aqueles profissionais que declararam não utilizar a estratégia ( $\mathrm{N}=38$ ). } \\
\hline \multicolumn{3}{|c|}{$\begin{array}{l}{ }^{4} \mathrm{~A} \text { questão sobre outras estratégias utilizadas para avaliar a criança, foi direcionada apenas aos profissionais que declararam não usar a estratégi } \\
\text { para atendimento das crianças e eles podiam dar mais de uma resposta }(\mathrm{N}=57) \text {. }\end{array}$} \\
\hline
\end{tabular}

\section{DISCUSSÃO}

A predominância de profissionais do sexo feminino na ESF é uma característica da força de trabalho em saúde na maioria dos estados brasileiros, conforme estudos realizados em diferentes cidades do país ${ }^{(13-14)}$.

O tempo relativamente curto de conclusão do curso de graduação dos profissionais que atuavam na ESF também já foi observado em outros estudos tais como o realizado com enfermeiros do Estado do Rio de Janeiro(15) e do Maranhão(16).

A graduação recente pode ser fator positivo para a consolidação das estratégias de atenção na saúde da família, inclusive a AIDPI, especialmente pelas mudanças ocorridas nos currículos das profissões da área da saúde, no sentido de buscar uma formação mais generalista, possibilitando aos estudantes o desenvolvimento de competências e habilidades para intervenções eficientes nos mais variados aspectos do processo saúde-doença dos indivíduos e comunidade. Além disso, a AIDPI vem sendo inserida como conteúdo nos cursos de graduação de medicina e enfermagem no país há mais de 15 anos $^{(12)}$.

No que se refere à pós-graduação, o perfil dos profissionais estudados é semelhante ao apresentado pelos que atuavam na ESF no município de Santa Cruz do Sul/RS e Serra/ES ${ }^{(13-14)}$. Esse perfil revela o interesse dos profissionais em aperfeiçoarem seu desempenho, além de ser uma forma de manter-se no emprego devido aos vínculos empregatícios instáveis aos quais estão submetidos. Ademais, o perfil de profissionais sanitaristas 
evidenciado no presente estudo pode ser entendido como comprometimento com o aperfeiçoamento do respaldo científico para o trabalho na ESF.

Quanto ao tempo de atuação dos profissionais na ESF, sabe-se que o tempo de permanência na unidade e a experiência profissional podem influenciar no desenvolvimento do trabalho e no estabelecimento de vínculo com a comunidade, aspecto fundamental para as ações realizadas na ESF, especialmente àquelas voltadas à criança, que necessitam da adesão da família para o acompanhamento da saúde infantil.

O estabelecimento de vínculo permite a corresponsabilização pela saúde ao longo do tempo por meio da construção de relações de confiança e afetividade entre os profissionais de saúde e a comunidade ${ }^{(17)}$. No entanto, essa construção demanda tempo. Nesse sentido, um tempo maior de atuação dos profissionais em uma mesma unidade é fundamental para que estratégias como a AIDPI alcancem os seus objetivos.

Desde 1998 o Ministério da Saúde do Brasil iniciou uma atuação mais próxima junto às universidades para que os conteúdos sobre os programas e estratégias governamentais, inclusive a AIDPI, fossem incorporados nos currículos de graduação em enfermagem e medicina, com o intuito de direcionar a formação dos profissionais às diretrizes políticas atuais, especialmente, voltadas à integralidade da atenção e promoção da saúde.

Apesar disso, estudo que avaliou o ensino da AIDPI nos cursos de graduação em enfermagem no Brasil, apontou que apenas $2 / 3$ dos referidos cursos incorporaram a AIDPI como conteúdo teórico em seus currículos, sendo que apenas $50 \%$ das faculdades pesquisadas incluíram atividades práticas da AIDPI no ensino de saúde da criança. O mesmo estudo apontou ainda que somente 39,4\% dos docentes eram capacitados em AIDPI ${ }^{(18)}$. Essa situação pode interferir na decisão de inserir este conteúdo nas atividades dos cursos de graduação.

Ainda no que diz respeito à inserção da AIDPI nos cursos de graduação, resultados de pesquisa com enfermeiros egressos da Escola de Enfermagem da Universidade de São Paulo mostrou que, os mesmos, além de reconhecerem a importância da aprendizagem da estratégia durante a graduação, apontam a necessidade de integrar todas as disciplinas que contemplam esse conteúdo, de modo a otimizar a carga-horária para que tal conteúdo seja melhor aproveitado ${ }^{(11)}$.

O sucesso da implantação e implementação da AIDPI depende, igualmente, do envolvimento das universidades e dos educadores, uma vez que a capacitação durante a graduação permite que os profissionais entrem no mercado de trabalho melhor qualificados e tornem-se agentes multiplicadores da estratégia para a comunidade e equipe.

No que se refere à capacitação dos profissionais em AIDPI, após o término da graduação, os resultados desse estudo mostraram que a maioria dos profissionais não fez nenhum tipo de curso/treinamento. Por sua vez, pesquisa que avaliou o conhecimento dos enfermeiros da ESF acerca da AIDPI, em Imperatriz/MA constatou que mais da metade dos participantes era capacitado na estratégia AIDPI ${ }^{(16)}$. Cabe ressaltar que a capacitação dos profissionais de saúde em AIDPI foi iniciada a partir de 1996, incluindo médicos e enfermeiros em uma atuação conjunta nos serviços de saúde. No entanto, a partir dos anos 2000 houve redução na continuidade das capacitações de forma sistematizada, já que a temática passou a ser incluída nos cursos de graduação de enfermagem e medicina. De acordo com gestores de saúde e facilitadores da estratégia AIDPI de Fortaleza/CE, o governo brasileiro tem diminuído os investimentos nas capacitações da estratégia, apesar de sua importância para a saúde infantil(12). 
O treinamento na estratégia AIDPI pode melhorar as habilidades, conhecimento e confiança dos profissionais. Estudo que avaliou o desempenho dos profissionais de saúde após o treinamento em AIDIP em Benin, evidenciou que esses foram mais propensos a classificar corretamente as doenças infantis, precrever os medicamentos, acompanhar a vacinação de crianças e aconselhar as famílias em relação à nutrição adequada e administração de terapias orais(3).

Pesquisa que avaliou a percepção dos estudantes de enfermagem sobre a utilização da AIDPI em Omã, na península Arábica, após capacitação e aplicação de um questionário, apontou que, para os estudantes, o treinamento sobre AIDPI foi benéfico à formação profissional e os mesmos pretendem aplicar os conhecimentos e habilidades relacionados à estratégia na futura prática clínica(19).

A utilização parcial da AIDPI foi evidenciada em alguns estudos realizados no Brasil. Destaca-se, nesta oportunidade, a incorporação da estratégia AIDPI na prática dos enfermeiros egressos da Escola de Enfermagem da Universidade de São Paulo (EEUSP), em que os profissionais estudados aplicavam parcialmente a estratégia e de forma restrita ao módulo de avaliação(11).

Igualmente ao exposto, pesquisa que analisou a prática dos enfermeiros e médicos na implementação da AIDPI em USF da mesma cidade onde foi desenvolvida a presente investigação, revelou utilização parcial, apontando que os profissionais aplicavam os aspectos recomendados pela metodologia, no entanto, sem a sistematização e uso dos instrumentos preconizados ${ }^{(20)}$. Cabe destacar que esses resultados são decorrentes de pesquisa qualitativa com um número pequeno de profissionais do Estado e da capital.

Na presente pesquisa, a falta de capacitação configurou-se como principal motivo para a ausência da utilização da estratégia por quase metade dos profissionais participantes. No entanto, muitas vezes, a capacitação dos profissionais em AIDPI não é suficiente para garantir uma atenção satisfatória em face de situações comuns de agravos como desidratação, infecção de ouvido aguda e desnutrição grave, conforme mostrou resultados de estudo que identificou o conhecimento dos enfermeiros da atenção básica sobre a AIDPI(16).

No que diz respeito às outras estratégias utilizadas pelos profissionais para avaliar a criança durante a consulta, a que mais se destacou foi a avaliação de crescimento e desenvolvimento, que parece ser entendida pelos profissionais como ação desvinculada da AIDPI. Essa compreensão equivocada também foi observada no estudo realizado anteriormente em Cuiabá/MT, Brasil(20). A AIDPI prevê em sua metodologia de atendimento todas as estratégias e programas mencionados pelos participantes da pesquisa, uma vez que a criança deve ser avaliada e classificada para a presença dos sinais e sintomas das doenças prevalentes ou outros agravos e encaminhada para os serviços de referência, caso necessário, além de ter o seu crescimento e desenvolvimento e situação vacinal avaliados. Outra ação prevista se refere à orientação da mãe ou responsável quanto aos cuidados com a criança em domicílio.

Apesar de questionamentos internacionais sobre a utilidade da estratégia AIDIP, nos últimos anos não houve revisão de sua implementação no Brasil, assim como em países como a Africa do Sul(9). Essa situação evidencia a necessidade de retomada dos investimentos governamentais para revitalização e fortalecimento da estratégia, com vistas a solucionar as falhas e dificuldades na implementação, em especial na capacitação dos profissionais de saúde, bem como, a priorização da AIDIP na agenda politica de saúde da criança dos estados e municipios brasileiros. 
A presente pesquisa possui limitações próprias do estudo descritivo, além de apresentar apenas a informação dos profissionais, sem a observação da prática. Contudo, os resultados poderão subsidiar o planejamento de políticas locais e chamar a atenção dos gestores e profissionais, sobre a importância da utilização da estratégia AIDIP para a saúde infantil, bem como subsidiar investimentos em outras pesquisas sobre a temática, tendo em vista a sua relevância para o cuidado da criança.

\section{CONCLUSÃO}

Os resultados mostraram que $44,6 \%$ dos profissionais participantes da pesquisa relataram utilizar parcialmente a estratégia AIDPI, sendo que o elemento mais utilizado por eles foi a avaliação do crescimento, desenvolvimento e da anemia. O principal motivo alegado para a ausência da utilização da AIDPI foi a falta de capacitação. A estratégia de atenção mais empregada no atendimento da criança, por aqueles que não a utilizam, foi a avaliação de crescimento e desenvolvimento, demonstrando o desconhecimento dos profissionais sobre a abrangência da AIDPI.

Os resultados encontrados na presente pesquisa evidenciam a necessidade de repensar a implementação e a utilização da AIDPI na capital e nas demais cidades do Estado, uma vez que esta produz impacto positivo na morbimortalidade infantil e em outros indicadores de saúde. Considerando que Cuiabá é referência na atenção à saúde da criança e mesmo assim apresentou limitações no uso da estratégia, possivelmente nos demais municipios da região essa situação seja semelhante. Além disso, os achados impulsionam a reflexão acerca da prática dos profissionais responsáveis pelo atendimento da criança e das práticas de gestão, principalmente no que tange à articulação de todas as estratégias de atendimento infantil com vistas à integralidade.

Ademais, os resultados reforçam a necessidade de mobilizar os gestores para a relevância da capacitação e educação continuada dos profissionais da saúde que atuam junto a criança, para maior qualificação das ações desenvolvidas na atenção integrada e integral à saúde da criança.

Apesar dos resultados da presente pesquisa serem idênticos aos encontrados em outras regiões do país, trazem avanços para a produção sobre AIDPI na região estudada, diante da escassez de pesquisas sobre o tema. Recomenda-se ampliar a pesquisa para outros municípios do Estado, para se ter um panorama mais ampliado da realidade sobre a estratégia na atenção primária. O estudo sinaliza também a necessidade de novos investimentos em pesquisa, em especial, estudos observacionais e analíticos que mostrem a situação atual da implementação da estratégia no país.

\section{REFERÊNCIAS}

1. Organização Pan-Americana da Saúde. Estratégia e Plano de Ação para a saúde integral na infância [Internet]. Washington, DC: OPAS, 2013 [acesso em: 27 nov. 2018]. Disponível em: https://www.paho.org/hq/dmdocuments/2013/ICH-portugues-final.pdf. 2. Ministério da Saúde, Secretaria de Atenção à Saúde, Departamento de Ações Programáticas e Estratégicas. Manual AIDPI neonatal [Internet]. Brasília: Ministério da Saúde, 2014 [acesso em: 27 nov. 2018]. Disponível em: http://portalarquivos.saude.gov.br/images/pdf/2016/fevereiro/03/Manual-Aidpi-corrigido-.pdf.

3. Steinhardt LC, Onikpo F, Kouamé J, Piercefield E, Lama M, Deming MS, et al. Predictors of health worker performance after Integrated Management of Childhood IIIness training in Benin: a cohort study. BMC Health Serv Res [Internet]. 2015 [acesso em: 27 nov. 2018];15:276. Disponível em: https://doi.org/10.1186/s12913-015-0910-4. 
4. Kiplagat A, Musto R, Mwizamholya D, Morona D. Factors influencing the implementation of integrated management of childhood illness (IMCI) by healthcare workers at public health centers \& dispensaries in Mwanza, Tanzania. BMC Public Health [Internet]. 2014 [acesso em: 27 nov. 2018];14:277. Disponível em: https://doi.org/10.1186/1471-2458-14-277.

5. Febir LG, Baiden FE, Agula J, Delimini RK, Akpalu B, Tivura M, et al. Implementation of the integrated management of childhood illness with parasitological diagnosis of malaria in rural Ghana: health worker perceptions. Malar J [Internet]. 2015 [acesso em: 27 nov. 2018];14:174. Disponível em: https://doi.org/10.1186/s12936-015-0699-y.

6. Mayhew M, Ickx P, Newbrander W, Stanekzai H, Alawi SA. Long and short Integrated Management of Childhood IIIness (IMCI) training courses in Afghanistan: a cross-sectional cohort comparison of post-course knowledge and performance. Int J Health Policy Manag [Internet]. 2015 [acesso em: 27 nov. 2018];4(3):143-52. Disponível em: https://doi.org/10.15171/ijhpm.2015.17. 7. Mupara LU, Lubbe JC. Implementation of the Integrated Management of Childhood Illnesses strategy: challenges and recommendations in Botswana. Glob Health Action [Internet]. 2016 [acesso em: 27 nov. 2018];9(1):29417. Disponível em: https://doi.org/10.3402/gha.v9.29417.

8. United Nations Children's Fund. Levels \& Trends in Child Mortality. Report 2014 [Internet]. Nova York (USA): UNICEF, 2015 [acesso em: 27 nov. 2018]. Disponível em: https://www.unicef.org/media/files/Levels and_Trends in_Child_Mortality_2014.pdf. 9. Fick C. Twenty years of $\mathrm{IMCl}$ implementation in South Africa: accelerating impact for the next decade. South African Health Review [Internet]. 2017 [acesso em: 27 nov. 2018];2017(1):207-14. Disponível em:

https://journals.co.za/content/journal/10520/EJC-c84c6d8c3.

10. Amaral JJF, Victora CG, Leite AJM, Cunha AJLA. Implementação da estratégia Atenção Integrada às Doenças Prevalentes na Infância no Nordeste, Brasil. Rev Saude Publica [Internet]. 2008 [acesso em: 27 nov. 2018];42(4):598-606. Disponível em: https://doi.org/10.1590/S0034-89102008000400004.

11. Higuchi CH, Fujimori E, Cursino EG, Chiesa AM, Veríssimo MDLOR, Mello DF. Atenção Integrada às Doenças Prevalentes na Infância (AIDPI) na prática de enfermeiros egressos da USP. Rev Gaucha Enferm [Internet]. 2011 [acesso em: 27 nov.

2018];32(2):241-7. Disponível em: https://doi.org/10.1590/S1983-14472011000200005.

12. Borda-Olivas A, Palma-Ruiz M, Amaral JJF. Percepciones de gestores de salud y facilitadores de la estrategia Atención Integrada a las Enfermedades Prevalentes (AIEPI) en una zona del Noreste de Brasil y Perú. Interface (Botucatu) [Internet]. 2017 [acesso em: 27 nov. 2018];21(63):933-43. Disponível em: https://doi.org/10.1590/1807-57622016.0718.

13. Moreira IJB, Horta JA, Duro LN, Borges DT, Cristofari AB, Chaves J, et al. Perfil sociodemográfico, ocupacional e avaliação das condições de saúde mental dos trabalhadores da Estratégia Saúde da Família em um município do Rio Grande do Sul, RS. Rev Bras Med Família e Comunidade [Internet]. 2016 [acesso em: 27 nov. 2018];11(38):1-12. Disponível em:

https://doi.org/10.5712/rbmfc11(38)967.

14. Lima EFA, Sousa AI, Primo CC, Leite FMC, Souza MHN, Maciel ELN. Perfil socioprofissional de trabalhadores de equipes saúde da família. Rev enferm UERJ [Internet]. 2016 [acesso em: 27 nov. 2018];24(1):e9405. Disponível em:

https://doi.org/10.12957/reuerj.2016.9405.

15. Faria MGA, Acioli S, Gallasch CH. Perfil de enfermeiros fluminenses da estratégia de saúde da família participantes de um curso de especialização. Enfermagem em Foco [Internet]. 2016 [acesso em: 27 nov. 2018];7(1):52-5. Disponível em:

https://doi.org/10.21675/2357-707X.2016.v7.n1.667.

16. Matos DHA, Martins TS, Fernandes MNF. ADIPI: conhecimento dos enfermeiros da Atenção Básica no interior do Maranhão. Journal of Health Sciences [Internet]. 2016 [acesso em: 27 nov. 2018];18(4):229-34. Disponível em:

http://www.pgsskroton.com.br/seer/index.php/JHealthSci/article/view/3629.

17. Ministério da Saúde, Secretaria de Atenção a Saúde, Departamento de Atenção Básica. PMAQ: Programa de Melhoria do Acesso e da Qualidade. Manual instrutivo para as equipes de Atenção Básica (Saúde da Família, Saúde Bucal e Equipes Parametrizadas) e NASF [Internet]. Brasília: Ministério da Saúde, 2013 [acesso em: 27 nov. 2018]. Disponível em: http://189.28.128.100/dab/docs/portaldab/publicacoes/manual instrutivo PMAQ AB2013.pdf.

18. Fujimori E, Higuchi CH, Cursino EG, Veríssimo MDLOR, Borges ALV, Melo DF et al. Teaching of the Integrated Management of Childhood Illness strategy in undergraduate nursing programs. Rev Lat Am Enfermagem [Internet]. 2013 [acesso em: 27 nov. 2018];21(3):655-62. Disponível em: https://doi.org/10.1590/S0104-11692013000300002.

19. Al-Araimi FA, Langrial SU. A Hypothetical Model to Predict Nursing Students' Perceptions of the Usefulness of Pre-Service Integrated Management of Childhood Illness Training. Sultan Qaboos Univ Med J [Internet]. 2016 [acesso em: 27 nov. 2018];16(4):e469-74. Disponível em: https://doi.org/10.18295/squmj.2016.16.04.011.

20. Boralho LLC, Corrêa LZM, Silva MA. Atenção Integrada às Doenças Prevalentes na Infância (AIDPI) em Cuiabá, MT: a prática dessa estratégia em unidades de saúde da família. Nursing (São Paulo). 2010;13(145):241-245. 